\title{
Macrophages and cellular immunity in Drosophila melanogaster
}

\author{
Katrina S. Gold ${ }^{2}$ and Katja Brückner ${ }^{1,2,3,4}$
}

${ }^{1}$ Eli and Edythe Broad Center of Regeneration Medicine and Stem Cell Research

${ }^{2}$ Department of Cell and Tissue Biology

${ }^{3}$ Cardiovascular Research Institute, University of California San Francisco, San Francisco, CA

${ }^{4}$ Corresponding Author:

35 Medical Center Way

San Francisco, CA 94143-0669

E-mail: katja.brueckner@ucsf.edu 


\begin{abstract}
The invertebrate Drosophila melanogaster has been a powerful model for understanding blood cell development and immunity. Drosophila is a holometabolous insect, which transitions through a series of life stages from embryo, larva and pupa to adulthood. In spite of this, remarkable parallels exist between Drosophila and vertebrate macrophages, both in terms of development and function. More than $90 \%$ of Drosophila blood cells (hemocytes) are macrophages (plasmatocytes), making this highly tractable genetic system attractive for studying a variety of questions in macrophage biology. In vertebrates, recent findings revealed that macrophages have two independent origins: self-renewing macrophages, which reside and proliferate in local microenvironments in a variety of tissues, and macrophages of the monocyte lineage, which derive from hematopoietic stem or progenitor cells. Like vertebrates, Drosophila possesses two macrophage lineages with a conserved dual ontogeny. These parallels allow us to take advantage of the Drosophila model when investigating macrophage lineage specification, maintenance and amplification, and the induction of macrophages and their progenitors by local microenvironments and systemic cues. Beyond macrophage development, Drosophila further serves as a paradigm for understanding the mechanisms underlying macrophage function and cellular immunity in infection, tissue homeostasis and cancer, throughout development and adult life.
\end{abstract}




\section{Introduction}

The blood cell system of the invertebrate Drosophila melanogaster comprises two myeloid lineages, which share highly conserved features with the vertebrate myeloid systems (Fig. 1). Unlike vertebrates, Drosophila lacks both a lymphoid system and red blood cells for oxygen transport, the latter instead being achieved by an extensive tracheal system. Drosophila largely relies on innate immunity, encompassing both a humoral response of antimicrobial peptide expression, and cellular responses of phagocytosis and encapsulation. In Drosophila, the major class of blood cells, or hemocytes, are plasmatocytes, which are considered equivalent to vertebrate macrophages. At every developmental stage, aside from the early embryo, more than $90 \%$ of all hemocytes are plasmatocytes [1-3], which have important functions during animal development, and in response to infection, tissue damage, and tumor growth.

\section{Macrophage lineages and development in Drosophila}

\subsection{Self-renewing macrophages of embryonic origin}

Recent work has shown that, in vertebrates, there are two developmentally independent lineages of macrophages [4-9]. They derive (1) from erythro-myeloid progenitors (EMPs) of the yolk sac $[10,11]$ and (2) from hematopoietic stem and progenitor cells (HSPCs) of the bone marrow, via differentiation into monocytes [12, 13] (Fig.1). EMP-derived macrophages colonize a multitude of organs during development and continue to self-renew in local microenvironments; they are therefore known as tissue-resident macrophages [8, 14-16]. Just like in vertebrates, Drosophila has a lineage of macrophages (plasmatocytes) that colonize tissues and self-renew in local microenvironments [17-19]. These macrophages originate from the procephalic (head) 
mesoderm of the embryo, which gives rise to a defined number of blood cell progenitors, also called prohemocytes [3] (Fig. 1, 2). More than 90\% of these prohemocytes differentiate into 600 plasmatocytes, which have macrophage-like roles in the removal of pathogens and apoptotic cells [3], and the deposition of extracellular matrix during development [20]. A small percentage of embryonic prohemocytes develop into crystal cells, a specialized blood cell type that catalyzes melanization reactions in response to wounding and pathogen invasion [21], and shows analogies to vertebrate granulocytes.

Many studies have dissected the factors that determine the fate of prohemocytes and promote their differentiation into plasmatocytes or other blood cell types. For example, several highly conserved transcription factors specify blood cell lineages during Drosophila embryogenesis. The GATA factor Serpent (Srp) is both necessary and sufficient for hemocyte specification in the embryonic mesoderm [22], acting upstream of the transcription factors Lozenge (Lz), Glial cells missing (Gcm) and U-shaped (Ush) [21, 23]. Gcm [24, 25] and Gcm2 [26] have redundant roles in specifying the plasmatocyte lineage in the embryo, while the Runx protein Lz [21], which is the Drosophila orthologue of Acute Myeloid Leukemia-1, is required for crystal cell specification. U-shaped, a zinc finger Friend of GATA (FOG) protein, acts to suppress crystal cell formation [23, 27]. The regulation and function of these transcription factors are reviewed in more detail elsewhere [19, 28, 29].

Plasmatocytes born in the Drosophila embryo colonize local microenvironments in the larva. This process is reminiscent of the colonization of fetal liver by EMPs in vertebrates, and the subsequent colonization of multiple organs, including brain, lung, skin, heart, and pancreas, by EMP-derived tissue macrophages $[10,11,15,16,30,31]$. In particular, Drosophila plasmatocytes form sessile, or resident, clusters in specific areas of the gastrointestinal system 
(proventriculus) [32] and microenvironments in the larval body wall (Hematopoietic Pockets) that form a segmentally repeated and terminal segment pattern [17, 18] (Fig. 1, 2). This population of plasmatocytes shows high rates of self-renewal, leading to a $>30$-fold expansion of the macrophage pool, from about 300 cells in the $1^{\text {st }}$ instar to around 10,000 in the late $3^{\text {rd }}$ instar $[33,34]$. While in the $1^{\text {st }}$ larval instar nearly all hemocytes are resident, an increasing number of hemocytes is found in circulation from the late $2^{\text {nd }}$ larval instar onward, forming a steady state with the resident hemocyte population. Drosophila resident plasmatocytes can also give rise to two other types of blood cells: crystal cells $[35,36]$ and, under immune challenge, lamellocytes [37], a type of hemocyte specialized for the encapsulation of large foreign bodies, such as parasitoid wasp eggs. This suggests that at least some, if not all, plasmatocytes have lineagerestricted progenitor capacity, underscoring further parallels with vertebrate EMPs [31]. Several studies have reported distinct subpopulations of plasmatocytes with varying combinations [37, 38], and quantitative expression differences [17], of commonly used plasmatocyte "markers" such as Hemolectin [39], Peroxidasin [40], P1 (Nimrod C1) [41], Croquemort [42], Eater [43], and the pan-hemocyte marker Hemese [44]. This favors the idea that specialized subsets of plasmatocytes exist, which could reflect distinct functional capabilities of these cells.

Recent lineage tracing and live imaging experiments [17,35] have left little room for a scenario in which undifferentiated progenitors would give rise to the resident and circulating hemocytes of the Drosophila larva, with the exception of the Lymph Gland (see Section 1.2 below). Nevertheless, a small fraction of potentially undifferentiated, Wingless-positive Hemolectinnegative cells has been reported among the resident/circulating hemocyte population [45], yet their potential to expand and contribute to the blood cell pool remains to be investigated.

In the Drosophila Hematopoietic Pockets, sensory neuron clusters of the peripheral nervous 
system (PNS) serve as an inductive microenvironments for plasmatocytes/macrophages, linking environmental sensory inputs to the control of the macrophage pool ([17] and Brückner lab, in revision). Activin- $\beta$ produced by local neurons promotes plasmatocyte proliferation and adhesion (Brückner lab, in revision). This is consistent with ablation studies showing that the PNS provides functional support to macrophages, promoting both their survival and localization [17]. The PNS is also known to innervate the proventriculus [46], suggesting further connections between the nervous system and resident macrophage populations. In vertebrates, the nature and regulation of tissue macrophage local microenvironments remain unknown. However, the anatomical juxtaposition of self-renewing macrophages and local populations of peripheral neurons, such as observed in the skin [47, 48] heart [49-51], and pancreas [52], suggest that similar regulatory relationships may exist.

Other components of the Drosophila Hematopoietic Pockets may also have roles in regulating Drosophila self-renewing macrophages. Larval muscle layers, which line the internal side of the Hematopoietic Pockets and on which plasmatocytes reside, are the sites of JAK/STAT signaling after parasitoid wasp infection. Interestingly, this signaling activity is required for mounting a cellular immune response against the parasite, and seems important for the mobilization of resident plasmatocytes into circulation, and their differentiation into lamellocytes [53].

The Hematopoietic Pockets of Drosophila also contain clusters of liver-like oenocytes [17, 18, 54], evoking parallels with the localization of vertebrate EMPs and other blood cell progenitors to the fetal liver during development $[10,31,55]$, and the residence of Kupffer cells (selfrenewing macrophages) in the liver throughout the vertebrate lifespan $[15,16]$. However, the question of potential regulatory roles requires further investigation in Drosophila, as initial 
studies based on oenocyte fate suppression have failed to detect a functional correlation between oenocytes and plasmatocyte localization [17].

Self-renewing macrophage populations in vertebrates have specific identities, based on their tissue of residence $[56,57]$. Tissue macrophage populations play important roles in human disease and have started to become the focus of therapeutic interventions, as exemplified by pulmonary macrophage transplantation [58]. Thus, understanding the role of local microenvironments in the regulation of progenitors and self-renewing macrophages is an important field of study that may provide a new interface, and molecular targets, for clinical therapies and prevention.

\subsection{Progenitor-derived macrophages of the Lymph Gland}

The second lineage of Drosophila macrophages derives from a hematopoietic organ, the Lymph Gland (LG), largely through a progenitor-based mechanism (Fig. 1, 2). The Lymph Gland develops during larval stages, but it arises earlier, from an independent embryonic mesodermal anlage of the same origin as the Drosophila heart-like organ, or dorsal vessel [2, 59-61]. The origin of Drosophila Lymph Gland progenitors from cardiogenic mesoderm echoes the origin of some vertebrate hematopoietic stem cells (HSCs) from a hemangioblast progenitor in the primitive streak, and HSCs from hemogenic endothelium of the aorta and other major arteries [29, 55, 62-64]. Prohemocytes of the Drosophila Lymph Gland mature from the mid-2nd larval instar onward, giving rise to an estimated 2000-3000 blood cells under non-immune challenged conditions. More than $90 \%$ of these cells are plasmatocytes, and the remainder consists of small fractions of crystal cells and lamellocytes [2, 29, 60]. By analogy to vertebrate macrophages originating from HSPCs, most Lymph Gland plasmatocytes derive from undifferentiated 
progenitors [60, 65]. In addition, differentiated Lymph Gland plasmatocytes undergo a relatively short phase of self-renewal, mainly in the $3^{\text {rd }}$ instar larva $[60,66,67]$. A distinct population of intermediate progenitors that show combinations of prohemocyte and plasmatocyte markers [68, 69], or lack prohemocyte and plasmatocyte markers [70], and are more proliferative than other cells, were reported [69, 70]; these cells are thought to contribute to the pool of Lymph Gland plasmatocytes. Thus Lymph Gland plasmatocytes show similarities to HSPC-derived macrophages of the monocyte lineage, which undergo limited proliferation, particularly in response to immune challenges [71, 72].

The Lymph Gland is organized into several pairs of lobes located at the anterior end of the dorsal vessel. The primary lobes are functionally regionalized into an undifferentiated medullary zone containing progenitors, sometimes distinguished by marker expression as pre-prohemocytes and prohemocytes, and a differentiated cortical zone [60]. By 12 hours after puparium formation, all hemocytes have differentiated and the Lymph Gland disintegrates, releasing its blood cells [73].

A large body of work has contributed to our understanding of Lymph Gland hemocyte differentiation, which is regulated by both local and systemic signals. Several reports converge on a key role for the Posterior Signaling Center (PSC), which comprises a small group of cells at the posterior end of the primary lobe. The PSC has been proposed to act as supportive microenvironment, or niche, that maintains Lymph Gland prohemocytes in an undifferentiated state [66, 74]. It has been reported that PSC cells send out several molecular cues to regulate the differentiation state of the Lymph Gland, including Serrate (Ser), Hedgehog (Hh), Wingless (Wg, Wnt), Decapentaplegic (Dpp, BMP) and Pvf1 (PDGF/VEGF-related factor) [59, 66, 68, 74, 75]. A recent study has challenged the roles of the PSC and Hedgehog signaling in maintaining progenitor maintenance, although it did confirm PSC function in inducing lamellocyte 
differentiation upon parasitization [76]. Serrate expression and the consequent activation of Notch signaling are required for crystal cell production [59] and maintaining the expression of Collier, a transcription factor which is highly expressed in PSC cells and has roles in the Lymph Gland response to wasp infestation [67, 76]. Wingless signaling has a dual role in the Lymph Gland. It controls PSC cell number cell-autonomously, and is also active in medullary zone prohemocytes, where it is required for progenitor maintenance [68]. Dpp antagonizes Wingless signaling in PSC cells, and is required cell autonomously to regulate the size of the niche [75]. Pvf1 is required to maintain prohemocytes, although interestingly this signal is not received by progenitors in the medullary zone, but by differentiating cells in the cortical zone. These cells in turn express the enzyme Adenosine Deaminase Growth Factor A (ADGF-A), which lowers extracellular adenosine levels. Low adenosine leads to reduced Protein Kinase A (PKA) activity and is thought ultimately to promote progenitor quiescence by stabilizing the active form of the transcription factor Cubitus interruptus (Ci) [77].

In addition, Lymph Gland hematopoiesis is regulated by many more inputs, including the Hippo [78 , 79], JAK/STAT [80, 81], Rel/NFאB-family related Toll [82] and FGFR [83] signaling pathways, as well as the heparan sulfate proteoglycan (and Perlecan orthologue) Trol [83, 84], the germ line differentiation factor Bag of Marbles (Bam) [85, 86], the zinc finger transcription factor Zfrp8 [87], the GATA factor Pannier [81], and the Polycomb group (PcG) gene multi sex combs ( $m x c)$ [88].

\subsection{Macrophages in the pupa and the adult}

Following their expansion and differentiation during larval development, the two Drosophila macrophage lineages persist through the pupal stage into the adult [89] (Fig. 2). At the transition 
to pupariation, plasmatocytes and other blood cell types are mobilized into circulation [17, 73], a process which is promoted by ecdysone signaling [90]. From this point onward, the two blood cell lineages intermix, and distinguishing embryonic and Lymph Gland hemocytes is, according to current methodology, only possible by lineage tracing. In the adult, plasmatocytes reside in or close to a number of tissues, including fat body (which regulates metabolism and immunity), heart, respiratory (tracheal) system, gut, peripheral nervous system, and ovaries ([38, 91-96] and Brückner lab in preparation). In addition, small numbers of crystal cells, but no lamellocytes, are present in the adult [2, 92, 97]. The differentiation status and plasticity of adult hemocytes is just beginning to be addressed, and may benefit from the development of Drosophila blood cell sublineage-specific antibodies and other hemocyte-specific research tools [41, 43, 98]. Recent findings indicate that functional subsets of adult macrophages have distinct physiological and signaling roles in immunity [99]. Notch signaling regulates crystal cell specification in the adult [92], which will be interesting to study in more detail in the future, given that transition from plasmatocyte to crystal cell fate is also known to occur during the embryonic and larval stages of development [27, 35, 36, 100]. Sex-specific factors may also influence the size and function of the adult blood cell pool [101] and further study will provide more insight into the long-term survival of Drosophila blood cells in males and females.

The proliferative capacity of adult plasmatocytes and other blood cells has been a matter of debate. A recent report claimed de novo production of hemocytes in the adult fly [92], whereas the majority of studies have not been able to obtain evidence of proliferation in adult hemocyte populations [2, 38, 97, 102], even under a range of immune-challenged conditions (Brückner lab in preparation). Indeed immunosenescence, involving a decline in both hemocyte number and 
phagocytic function, has been documented as adult flies age [101]. No evidence of homeostatic hemocyte maintenance has been obtained [102].

\section{Macrophage functions in Drosophila}

Macrophage functions in Drosophila include the removal of apoptotic cells during development, the production of extracellular matrix, and responses to immune invaders and damaged or aberrant tissue. Many of these aspects show close parallels with vertebrate systems, which have been reviewed in detail elsewhere [19, 29, 103, 104].

\subsection{Macrophage functions in the embryo}

In the Drosophila embryo, a major function of macrophages is the elimination of apoptotic cells [3], which is critical for development. Phagocytosis by Drosophila plasmatocytes requires scavenger receptors such as Croquemort (Crq) [105], Draper [106, 107], Eater [36, 108] and other Nimrod family proteins [109, 110], as well as adhesion molecules such as integrins [111]. In the embryo, phagocytosis by macrophages is essential for remodeling the central nervous system (CNS). Plasmatocytes phagocytose apoptotic neurons along the CNS midline, which ensures proper condensation of the nervous system [112, 113] and is required for embryonic survival. This has been demonstrated in Bicaudal-D and srp mutants, which lack embryonic hemocytes [22, 113, 114], as well as $c r q$ mutants and mutants of the receptor tyrosine kinase Pvr (PDGF/VEGF Receptor) [113], in which embryonic hemocytes undergo premature apoptotic death [115]. In the embryonic tracheal system, macrophages have a similar role in the elimination of apoptotic cells during tissue remodeling [116]. 
Embryonic plasmatocytes have important roles in the deposition of extracellular matrix (ECM) components and the production of ECM-associated molecules, including Collagen IV [20, 117, 118], Laminin [119], Tiggrin [120], Papilin [121], Peroxidasin [122, 123], and $\beta$ PS Integrin [124]. The importance of hemocyte-mediated sculpting of the ECM has been demonstrated in the context of nervous system development [125], the positioning of the renal (Malpighian) tubules [126], and the deposition of basal laminae surrounding internal organs such as the brain and gut [20].

Macrophage functions in the Drosophila embryo typically involve their regulated migration and invasion, such as their entry into the posterior end of the embryo at germband extension [3, 115, 127], or their infiltration of the nerve cord at the ventral midline [3, 124, 128]. Both systems have provided excellent opportunities for addressing the cellular and molecular mechanisms of macrophage migration and invasion, as reviewed comprehensively elsewhere [129-131].

The Drosophila embryo has also proved fruitful for the study of macrophage functions in response to injury, which is detailed thoroughly in many studies and reviews [40, 130, 132-135]. The Drosophila embryo has further been used as a model for septic injury (Fig. 3). At later stages, Drosophila embryos are competent to mount immune reactions, both through cellular mechanisms, involving phagocytosis by plasmatocytes [136], and humoral responses via the induction of antimicrobial peptide expression in the respiratory (tracheal) epithelium [137]. The competence of the respiratory epithelium to mount a humoral response is promoted by the steroid hormone ecdysone, which peaks at stage 12 of embryogenesis [137].

\subsection{Macrophage functions in the larva}


The larval stage is the critical phase for the expansion and adaptation of the immune cell pool. Environmental, metabolic, infection- and injury-related signals impinge on a plethora of signalling pathways that regulate the two larval myeloid systems, and in many cases lead them to mount a cellular immune response (see also above and [19]). Embryonically derived selfrenewing macrophages (sometimes called 'larval hemocytes') are located in resident clusters close to barrier epithelia, particularly in the Hematopoietic Pockets beneath the epidermis [17, 18], and around the proventriculus, an area of the gastrointestinal system that may act as a sink for bacteria and debris [32]. As the larva matures, increasing numbers of self-renewing plasmatocytes detach and enter into circulation [17, 34, 97], potentially monitoring the hemolymph for pathogens. Hemocytes of the Lymph Gland mature over the course of larval life, and are usually released into circulation only at the beginning of pupariation [60, 73, 97]. However, upon immune or injury challenge, both hemocyte lineages are mobilized into a cellular immune response (see below) (Fig. 3).

\subsubsection{Responses to sensory and metabolic stimuli}

Increasing evidence suggests that environmental sensory detection impacts the regulation of the two myeloid lineages in the Drosophila larva. Linking sensory inputs, which signal beneficial or adverse environmental life conditions, with the expansion of the immune cell pool may be an important safeguard for the animal to survive challenges, such as increased apoptotic cell death, or adapt to metabolic conditions. Environmental stimuli may be relayed to self-renewing macrophages through peripheral sensory neuron clusters in the Hematopoietic Pockets $[17,18]$ (Brückner lab in revision). At these locations, sensory neurons are in direct contact with hemocytes and link neuronal activity to Activin $\beta$ production, which promotes macrophage adhesion and proliferation (Brückner lab in revision). In the Lymph Gland, sensory inputs are 
linked to blood cell responses through systemic signals. Olfactory neurons in the CNS produce the neurotransmitter GABA, which signals systemically to the Lymph Gland, where it triggers calcium signaling and macrophage maturation [138].

Systemic signals also directly link the metabolic status of the animal with the regulation of the blood cell pool. For example, starvation drives the localization of plasmatocytes to the fat body [139], a fat-storing tissue with roles in metabolism and immunity. Changes in insulin signaling lead to the premature differentiation of Lymph Gland progenitors [69, 139-141] and similar effects are also triggered by starvation, detected by the amino acid transporter Slimfast [139]. Reactive Oxygen Species (ROS) levels in the Lymph Gland respond to metabolic stress and Tor pathway activity [69, 142], and excessive ROS production stimulates precocious differentiation of Lymph Gland hemocytes [143].

\subsubsection{Responses to parasitization}

In the Drosophila larva, a major model for studying cellular immunity is infestation by parasitoid wasps, such as Leptopilina boulardi $[97,144,145]$. In response to parasitization, self-renewing plasmatocytes mobilize rapidly into circulation from their resident sites, and differentiate into lamellocytes [37]. In a second wave response that occurs one or more days later [37], hemocytes of the Lymph Gland undergo a burst of proliferation, differentiate precociously, and are released into circulation, thus acting as an emergency reservoir of active blood cells in the larva [97, 144, 146]. Under these conditions, cell signaling from the Posterior Signaling Center is required to induce lamellocyte differentiation [74, 76]. Hemocyte proliferation in the Lymph Gland after a parasitic challenge requires the systemic steroid hormone ecdysone, explaining why $3^{\text {rd }}$ but not

$2^{\text {nd }}$ instar larvae can mount a Lymph Gland immune response [146]. The cellular immune 
response by hemocytes of both origins encompasses phagocytosis by plasmatocytes, encapsulation by lamellocytes, and melanization by crystal cells and lamellocytes [147],

These responses often depend on a relay of signals, either systemically and/or through other tissues. [148]. For example, communication between hemocytes and larval muscle cells that line the Hematopoietic Pockets has an important role in wasp egg encapsulation. Parasitization triggers circulating hemocytes to secrete the cytokines Unpaired 2 (Upd2) and Unpaired 3 (Upd3) that activate JAK/STAT signaling in somatic muscle, which is necessary for lamellocyte formation and wasp egg encapsulation [53]. However, JAK/STAT signaling alone is not sufficient to trigger encapsulation [53]; other inputs are required in this process, such as the activation of Toll signaling in the fat body [149], and pathways such as JNK signaling drive lamellocyte formation $[150,151]$, yet their route of relay remains to be investigated. Moreover, the systemic peptide Edin, which is expressed in the fat body upon wasp infestation, induces wasp egg encapsulation, as well as mobilization and expansion of plasmatocytes, but not their differentiation into lamellocytes [148]. Future investigation will show whether other tissues, such as the liver-like oenocytes [17-19] or other components of the Hematopoietic Pockets, may also have roles in relaying cellular and humoral immunity.

\subsubsection{Responses to infection and injury}

A large body of work on cellular immunity in the Drosophila larva has focused on intestinal infections. Here, hemocytes stimulate cellular and humoral immune reactions, the latter through the expression of antimicrobial peptides (AMPs), which are induced by the two major innate immune pathways of NFkB signaling, Toll and Imd [152]. Plasmatocytes in the larva function as phagocytic sentinels combating microbial infection, for example in response to gut infections induced by feeding on Serratia marcescens (S. marcescens), which causes an Imd pathway- 
dependent local response [153]. Hemocytes also act as important cellular relays, signaling to the fat body to coordinate immune responses across different tissues. For example, natural infection (feeding) by the Drosophila pathogen Erwinia carotovora carotovora (Ecc15) induces AMP expression in fat body, a response that is decreased in Domino mutants that lack blood cells [154]. Hemocytes are thought to act as a relay in a Nitric Oxide-induced systemic immune response to gram-negative infection, triggering Imd pathway activation in the fat body [155]. Similarly, hemocytes relay Ecc15-induced local stress signaling in the intestine, which is mediated by reactive oxygen species (ROS), to the fat body, where AMP expression is induced [156]. Defensin expression in the fat body depends on pathogen degradation in plasmatocytes, which requires the lysosomal protein Psidin [157]. Bacterial infection also triggers AMP expression in barrier epithelia, such as the respiratory system (trachea) [158], but the involvement of hemocytes in this response remains to be elucidated.

Using the Drosophila larva as a septic injury model has proved fertile ground for studying innate immune responses to infection [159-161]. Many of these studies have focused on the humoral immune response, but there is also a cellular component mediated by macrophages. Similarly to natural infections through the gut, septic injury leads to the upregulation of antimicrobial peptides in the larval fat body, and this response depends on a signal relayed by hemocytes through secretion of the Toll pathway ligand Spätzle [162].

Cellular responses against bacteria provide an opportunity to examine the phagocytic function of macrophages in more detail. Several studies have used larval hemocytes ex vivo to investigate the function of phagocytic receptors, such as the scavenger receptors Eater [108] and Nimrod C1 [109], and other proteins, such as the actin cytoskeleton regulators D-SCAR and Profilin, in the control of bacterial phagocytosis [163]. 
Aseptic injury triggers a cellular immune response that includes the mobilization of resident hemocytes, phagocytosis, and differentiation into lamellocytes, recapitulating many aspects of septic injury and parasitization [164]. Several studies have examined the cellular mechanisms of phagocytosis and encapsulation during aseptic injury [164, 165], and are reviewed in detail elsewhere [133, 135].

\subsubsection{Roles in ECM production and organ integrity}

Plasmatocytes in the larva continue to play important roles in the production of ECM, which is crucial for organogenesis and organ function. During larval development, hemocytes associate with the female gonad and secrete the ECM molecule Collagen IV (ColIV) [38]. These layers of ColIV are required during pupariation and adulthood to ensure proper molecular function of the germline stem cell niche, and in turn, germline stem cell number and homeostasis [38]. The possibility that macrophages regulate other stem cell microenvironments is tantalizing, and will be interesting to explore in the future.

\subsection{Macrophage functions in the pupa}

Drosophila undergoes pupariation and metamorphosis when ecdysone levels peak, signaling the end of larval development. Many larval tissues, which are often polyploid, are partially or completely replaced by adult structures that arise from sets of imaginal cells or discs (e.g. eye, wing, and leg discs) [166]. Drosophila macrophages have roles in the destruction and remodeling of these larval tissues. For example, at the onset of metamorphosis, plasmatocytes associate with larval fat body cells and facilitate their degradation, a process that is continued well into the first week of adult life [93]. Macrophages also participate in neuronal pruning at 
axons and dendrites [167], although a substantial part of this process may be mediated by epidermal cells and glia [168, 169]. Macrophages facilitate the remodelling of many other structures and organ systems, as exemplified by the maturation of wing discs, where hemocytes are required for the bonding of the dorsal and ventral wing regions [170].

In the pupa, macrophages show less resident cluster formation. Instead, they have been studied for their migratory and dynamic properties, which involve Integrin and other adhesion-related proteins [171], and are important for wound healing [172]. Macrophage motility and phagocytosis are also enhanced by ecdysone signaling [173]. Surprisingly, for the most part macrophages may not be essential during pupariation and adulthood. Animals with genetically ablated hemocytes are viable [174, 175], although developmental defects have been found at low penetrance $[174,176]$.

\subsection{Macrophage functions in the adult}

In the adult fly, plasmatocytes continue in their capacity as professional phagocytes, performing tissue repair [91, 177-179], immune surveillance and defense (Fig. 3). Anatomically, hemocytes in the adult fly are found in close proximity to the fat body and many surface epithelia, such as the respiratory (tracheal) epithelium and areas of the gastrointestinal system (Brückner lab in preparation and [91]) (Fig. 3). Clusters of hemocytes are also found in the dorsal abdomen around the heart [92, 94-96]. These accumulations are thought to monitor and clear the hemolymph of pathogens, and have been reported in Drosophila and other invertebrates, such as the mosquito Anopheles gambiae [96, 99, 180]. Classic invertebrate literature described these hemocyte clusters as 'invertebrate phagocytic organs' [181-183]. Indeed, recent publications on 
septic injury models have highlighted the correlation between bacterial and particle accumulations around the heart, and corresponding macrophage accumulations [94-96].

Many studies on the humoral immune response in adult Drosophila also report associated roles for macrophages. Oral infections with $S$. marcescens trigger a dual immune reaction, comprising a cellular immune response mediated by phagocytic plasmatocytes and a local intestinal antimicrobial response regulated by the Imd pathway [153]. Signaling interactions between hemocytes and other tissues enable a coordinated immune response to be mounted in the adult fly, as observed in the larva. For example, septic injury, or stimulation with the bacterial cell membrane component LPS (lipopolysaccharide), trigger expression of the cytokine ligand Upd3 in adult macrophages, which in turn activates JAK/STAT signaling in the fat body, leading to the upregulation of immune response genes [184]. Adult macrophages also respond to wounding and bacterial infection through other growth factors, as exemplified by subpopulations of hemocytes that express the BMP ligand Dpp or the Activin/TGF-beta ligand Dawdle [99]. Antimicrobial peptides are expressed in a variety of barrier epithelia in the adult fly, including Drosocin and Drosomycin in tracheal epithelia, Diptericin, Defensin and Attacin in the gut, Metchnikowin and Defensin in oral regions, Metchnikowin in renal tubules, and Cecropin, Drosomycin and Defensin in reproductive tracts $[185,186]$. It will be interesting to investigate possible links between these responses and macrophage signaling. Considering the typically short duration of antimicrobial peptide expression $[187,188]$, and recent findings on the dual role of hemocytes in humoral and cellular immunity [189], it appears likely that phagocytosis-mediated cellular immunity has an important role during the infection response in adults. Consistent with this hypothesis, inhibiting hemocyte function by expressing a bacterial toxin (ExoS) that suppresses phagocytosis leads to increased sensitivity to bacterial infection [190]. Further evidence of a role 
for cellular immunity in adult Drosophila comes from TM9SF4 nonaspanin mutant flies, which have phagocytosis-defective hemocytes yet a seemingly unaffected AMP response, and show increased lethality upon infection by gram-negative bacteria [191]. Blocking phagosome activity and bacterial degradation in adult flies leads to increased sensitivity to bacterial infection, as seen in mutants of full of bacteria, which encodes an ortholog of a HOPS complex subunit necessary for vacuolar fusion in yeast [96]. Adult macrophages require the phagocytic receptor Eater [108], which is also required in hemocytes at earlier developmental stages and has specificity for grampositive bacteria [36]. Loss of macrophages in the adult fly, induced either by targeted genetic ablation or specific mutant backgrounds (e.g. domino), results not only in a weakened immune response, but also in decreased long-term survival after bacterial infection [174, 175, 192]. This suggests that cellular immunity may have additional functions over the course of infection, not just in the short-term. However, alternative scenarios are possible, as it was recently shown that hemocyte ablation leads to a shift in inflammation status, with an upregulation of Toll signaling and downregulation of the Imd pathway [176]. Interestingly, adult macrophage responses may also be linked to environmental conditions. For example, phagocytosis and cellular immunity appear to be regulated by circadian inputs, yet melanization responses and humoral immunity are not, as evidenced by a model of infection with the bacterial pathogen Streptococcus pneumonia [193].

One controversial question in the field has been whether any kind of priming, or adaptive immune response, may exist in Drosophila. Some reports suggested such phenomena, either through unknown mechanisms or through alternatively spliced variants of the cell surface molecule DSCAM [188, 194]. However, future analysis will show whether additional mechanistic evidence for these phenomena can be obtained [195]. 


\subsection{Macrophage functions in damage-induced tissue regeneration}

Hemocytes promote damage-induced tissue recovery and tissue growth at various developmental stages. Under certain conditions, hemocytes not only promote the regeneration of tissues, but drive their pathological overproliferation. Similarly, macrophages in vertebrates play a host of vital roles in tissue repair and regeneration, stimulating proliferation of damaged tissue and causing hyperplasia or hypertrophy in some systems [196-199]. Drosophila has allowed to identify molecular mechanisms underlying these processes. For example, hemocytes play a key role in the recovery of UV- and JNK signaling-induced damage of eye imaginal disc epithelium that suffers apoptotic cell loss. Damaged tissue, through induction of the transcriptional regulator Schnurri (Shn), produces the PDGF/VEGF related ligand Pvf-1, which activates resident

plasmatocytes that in turn limit tissue loss [200]. During apoptosis-induced compensatory proliferation of imaginal epithelia, cells with elevated Caspase activity produce reactive oxygen species (ROS) that induce activation of resident plasmatocytes. These activated macrophages produce the inflammatory cytokine Tumor Necrosis Factor (TNF)/Eiger, which in turn triggers JNK signaling in the epithelial cells, leading to overproliferation [201]. In adult Drosophila, the regenerative response to infection- or stress-induced injury of the gut, which often is associated with dysplasia, [202, 203] depends both on the production of ROS by enterocytes [202, 204] and the active involvement of hemocytes [91]. Intestinal tissue damage attracts plasmatocytes and stimulates them to produce BMP, which in turn triggers intestinal stem cell proliferation, resulting in gut dysplasia [91].

\subsection{Macrophage functions in tumor biology}


Drosophila is increasingly being used as a model for cancer [205, 206]. Hemocytes participate both in the immune response against tumors, as well as the promotion of tumor growth. Hemocytes are recruited to neoplastic tumors, which are often sites of basement membrane disruption, and thus bear some similarities to non-healing wounds or tissue damage challenges [207-209]. Hemocytes adhere to epithelial tumors, and their numbers increase in response to tumor formation [208, 209] involving tumor-derived signals that stimulate JAK/STAT or Pvr signaling in hemocytes [208, 210]. Where hemocytes mount an immune attack against tumors, responses such as phagocytosis, induction of apoptosis, and melanization/encapsulation by crystal cells and lamellocytes, which is considered a functional equivalent to granulomas in vertebrates, are seen [207, 208, 210].

As in vertebrates [211-213], however, the effects of macrophage recruitment and inflammation on tumor biology vary, depending on the specific genetic background and microenvironment of the tumor, and this warrants extensive future research. For example, hemocytes associated with epithelial tumors express the inflammatory cytokine Eiger/TNF. In the case of Ras-transformed, scribble mutant tumors, activation of TNF signaling has a tumor-promoting effect [209]. In contrast, in a different epithelial tumor model based on discs large (dlg) mutants, TNF signaling acts to suppress tumors [210].

Mounting an anti-tumor response by macrophages can depend on the concerted action of multiple molecular mechanisms. In the Drosophila dlg tumor model, hemocytes not only secrete TNF but also Spätzle, the Toll pathway ligand. This results in a two-pronged tumor defense response: TNF signaling from hemocytes promotes tumor death directly, and Spätzle triggers a systemic immune response in the fat body, which acts in parallel to induce tumor cell apoptosis [210]. 
There are also reports of synergy between bacterial infections and oncogenic mutations, which together promote more severe gut dysplasia in response to tissue damage [214, 215]. The Imd and JNK signaling pathways mediate this interaction, but as of yet, no role for hemocytes has been demonstrated in the process. Thus, the complexities of the interactions between tumors, macrophages and cellular microenvironments are just beginning to be unraveled.

\section{Conclusions}

Drosophila melanogaster has become a diverse and versatile model to dissect the mechanisms of macrophage development and function. A large body of work has established high evolutionary conservation between this invertebrate and vertebrates, at the cellular and molecular level. Each developmental stage of Drosophila holds its own strengths for certain types of investigation, and in many cases their potential for experimental modeling is expected to grow even further in the future. Drosophila and its genetic toolkit allows us to investigate the mechanisms by which cellular microenvironments and long-range systemic signals coordinate communication between various tissues, ultimately shaping the development and adaptation of macrophages. It will further broaden our understanding of the innate cellular and humoral responses in infection and tissue development, homeostasis, regeneration and cancer.

\section{Acknowledgements}

K.B. thanks F. Geissmann for advice on hematopoietic waves and macrophage lineages in vertebrate systems, and E. J. V. Ramond for comments on the manuscript. We thank all members of the Brückner lab for discussion and feedback. This work was supported by a postdoctoral 
fellowship from the American Heart Association (to K.S.G.), and grants from the American

Cancer Society RSG DDC-122595, National Science Foundation 1326268, National Institutes of Health 1R01GM112083-01 and 1R56HL118726-01A1 (to K.B.). We apologize to authors whose work was not cited due to space constraints.

\section{References}

1. Rizki, T. and R. Rizki, The cellular defense system of Drosophila melanogaster. Insect Ultrastructure, 1984. 2: p. 579-604.

2. Rizki, T.M., The circulatory system and associated cells and tissues. In 'The Genetics and Biology of Drosophila', (M. Ashburner and T.R.F. Wright, Edts.), Academic Press, New York, 1978. 2b: p. 397-452.

3. Tepass, U., et al., Embryonic origin of hemocytes and their relationship to cell death in Drosophila. Development, 1994. 120(7): p. 1829-37.

4. van Furth, R. and M.M. Diesselhoff-den Dulk, Dual origin of mouse spleen macrophages. The Journal of experimental medicine, 1984. 160(5): p. 1273-83.

5. Herbomel, P., B. Thisse, and C. Thisse, Zebrafish early macrophages colonize cephalic mesenchyme and developing brain, retina, and epidermis through a M-CSF receptordependent invasive process. Developmental biology, 2001. 238(2): p. 274-88.

6. Ginhoux, F., et al., Fate mapping analysis reveals that adult microglia derive from primitive macrophages. Science, 2010. 330(6005): p. 841-5.

7. Schulz, C., et al., A lineage of myeloid cells independent of Myb and hematopoietic stem cells. Science, 2012. 336(6077): p. 86-90.

8. Hashimoto, D., et al., Tissue-resident macrophages self-maintain locally throughout adult life with minimal contribution from circulating monocytes. Immunity, 2013. 38(4): p. $792-804$.

9. Hoeffel, G., et al., Adult Langerhans cells derive predominantly from embryonic fetal liver monocytes with a minor contribution of yolk sac-derived macrophages. The Journal of experimental medicine, 2012.

10. Gomez Perdiguero, E., et al., Tissue-resident macrophages originate from yolk-sacderived erythro-myeloid progenitors. Nature, 2015. 518(7540): p. 547-51.

11. Hoeffel, G., et al., C-Myb(+) erythro-myeloid progenitor-derived fetal monocytes give rise to adult tissue-resident macrophages. Immunity, 2015. 42(4): p. 665-78.

12. Haniffa, M., V. Bigley, and M. Collin, Human mononuclear phagocyte system reunited. Seminars in cell \& developmental biology, 2015. 41: p. 59-69.

13. Swirski, F.K., I. Hilgendorf, and C.S. Robbins, From proliferation to proliferation: monocyte lineage comes full circle. Seminars in immunopathology, 2014. 36(2): p. 13748. 
14. Gomez Perdiguero, E. and F. Geissmann, Myb-independent macrophages: a family of cells that develops with their tissue of residence and is involved in its homeostasis. Cold Spring Harbor symposia on quantitative biology, 2013. 78: p. 91-100.

15. Davies, L.C., et al., Tissue-resident macrophages. Nature immunology, 2013. 14(10): p. 986-95.

16. Sieweke, M.H. and J.E. Allen, Beyond stem cells: self-renewal of differentiated macrophages. Science, 2013. 342(6161): p. 1242974.

17. Makhijani, K., et al., The peripheral nervous system supports blood cell homing and survival in the Drosophila larva. Development, 2011. 138: p. 5379-91.

18. Makhijani, K. and K. Brückner, Of blood cells and the nervous system: Hematopoiesis in the Drosophila larva. Fly, 2012. 6(4): p. 254-60.

19. Gold, K.S. and K. Brückner, Drosophila as a model for the two myeloid blood cell systems in vertebrates. Experimental hematology, 2014. 42(8): p. 717-727.

20. Martinek, N., et al., Haemocyte-derived SPARC is required for collagen-IV-dependent stability of basal laminae in Drosophila embryos. Journal of cell science, 2008. 121(Pt 10): p. 1671-80.

21. Lebestky, T., et al., Specification of Drosophila hematopoietic lineage by conserved transcription factors. Science, 2000. 288(5463): p. 146-9.

22. Rehorn, K.P., et al., A molecular aspect of hematopoiesis and endoderm development common to vertebrates and Drosophila. Development, 1996. 122(12): p. 4023-31.

23. Fossett, N., et al., The Friend of GATA proteins U-shaped, FOG-1, and FOG-2 function as negative regulators of blood, heart, and eye development in Drosophila. Proc Natl Acad Sci U S A, 2001. 98(13): p. 7342-7.

24. Bernardoni, R., V. Vivancos, and A. Giangrande, glide/gcm is expressed and required in the scavenger cell lineage. Dev Biol, 1997. 191(1): p. 118-30.

25. Jacques, C., et al., A novel role of the glial fate determinant glial cells missing in hematopoiesis. Int J Dev Biol, 2009. 53(7): p. 1013-22.

26. Alfonso, T.B. and B.W. Jones, gcm2 promotes glial cell differentiation and is required with glial cells missing for macrophage development in Drosophila. Dev Biol, 2002. 248(2): p. 369-83.

27. Fossett, N., et al., Combinatorial interactions of serpent, lozenge, and U-shaped regulate crystal cell lineage commitment during Drosophila hematopoiesis. Proc Natl Acad Sci U S A, 2003. 100(20): p. 11451-6.

28. Fossett, N. and R.A. Schulz, Functional conservation of hematopoietic factors in Drosophila and vertebrates. Differentiation, 2001. 69(2-3): p. 83-90.

29. Evans, C.J., V. Hartenstein, and U. Banerjee, Thicker than blood: conserved mechanisms in Drosophila and vertebrate hematopoiesis. Dev Cell, 2003. 5(5): p. 673-90.

30. Frame, J.M., K.E. McGrath, and J. Palis, Erythro-myeloid progenitors: "definitive" hematopoiesis in the conceptus prior to the emergence of hematopoietic stem cells. Blood cells, molecules \& diseases, 2013. 51(4): p. 220-5.

31. McGrath, K.E., et al., Distinct Sources of Hematopoietic Progenitors Emerge before HSCs and Provide Functional Blood Cells in the Mammalian Embryo. Cell reports, 2015. 11(12): p. 1892-904.

32. Zaidman-Remy, A., et al., The Drosophila larva as a tool to study gut-associated macrophages: PI3K regulates a discrete hemocyte population at the proventriculus. Developmental and comparative immunology, 2012. 36(4): p. 638-47. 
33. Sopko, R., et al., A systems-level interrogation identifies regulators of Drosophila blood cell number and survival. PLoS genetics, 2015. 11(3): p. e1005056.

34. Petraki, S., B. Alexander, and K. Bruckner, Assaying Blood Cell Populations of the Drosophila melanogaster Larva. Journal of visualized experiments : JoVE, 2015(105).

35. Leitao, A.B. and E. Sucena, Drosophila sessile hemocyte clusters are true hematopoietic tissues that regulate larval blood cell differentiation. eLife, 2015(in press).

36. Bretscher, A.J., et al., The Nimrod transmembrane receptor Eater is required for hemocyte attachment to the sessile compartment in Drosophila melanogaster. Biology open, 2015. 4(3): p. 355-63.

37. Markus, R., et al., Sessile hemocytes as a hematopoietic compartment in Drosophila melanogaster. Proc Natl Acad Sci U S A, 2009. 106(12): p. 4805-9.

38. Van De Bor, V., et al., Companion Blood Cells Control Ovarian Stem Cell Niche Microenvironment and Homeostasis. Cell reports, 2015. 13(3): p. 546-60.

39. Sinenko, S.A. and B. Mathey-Prevot, Increased expression of Drosophila tetraspanin, Tsp68C, suppresses the abnormal proliferation of ytr-deficient and Ras/Raf-activated hemocytes. Oncogene, 2004. 23(56): p. 9120-8.

40. Stramer, B., et al., Live imaging of wound inflammation in Drosophila embryos reveals key roles for small GTPases during in vivo cell migration. J Cell Biol, 2005. 168(4): p. 567-73.

41. Kurucz, E., et al., Definition of Drosophila hemocyte subsets by cell-type specific antigens. Acta Biol Hung, 2007. 58 Suppl: p. 95-111.

42. Franc, N.C., et al., Croquemort, a novel Drosophila hemocyte/macrophage receptor that recognizes apoptotic cells. Immunity, 1996. 4(5): p. 431-43.

43. Tokusumi, T., et al., New hemocyte-specific enhancer-reporter transgenes for the analysis of hematopoiesis in Drosophila. Genesis, 2009. 47(11): p. 771-4.

44. Kurucz, E., et al., Hemese, a hemocyte-specific transmembrane protein, affects the cellular immune response in Drosophila. Proc Natl Acad Sci U S A, 2003. 100(5): p. 2622-7.

45. Sinenko, S.A., et al., Genetic manipulation of AML1-ETO-induced expansion of hematopoietic precursors in a Drosophila model. Blood, 2010. 116(22): p. 4612-20.

46. Cognigni, P., A.P. Bailey, and I. Miguel-Aliaga, Enteric neurons and systemic signals couple nutritional and reproductive status with intestinal homeostasis. Cell metabolism, 2011. 13(1): p. 92-104.

47. Boulais, N. and L. Misery, The epidermis: a sensory tissue. European journal of dermatology : EJD, 2008. 18(2): p. 119-27.

48. Misery, L., Langerhans cells in the neuro-immuno-cutaneous system. Journal of neuroimmunology, 1998. 89(1-2): p. 83-7.

49. Brack, K.E., The heart's 'little brain' controlling cardiac function in the rabbit. Experimental physiology, 2015. 100(4): p. 348-53.

50. Pauza, D.H., et al., Morphology, distribution, and variability of the epicardiac neural ganglionated subplexuses in the human heart. The Anatomical record, 2000. 259(4): p. 353-82.

51. Pauziene, N., et al., Innervation of the rabbit cardiac ventricles. Journal of anatomy, 2016. 228(1): p. 26-46.

52. Razavi, R., et al., TRPV1+ sensory neurons control beta cell stress and islet inflammation in autoimmune diabetes. Cell, 2006. 127(6): p. 1123-35. 
53. Yang, H., et al., JAK/STAT signaling in Drosophila muscles controls the cellular immune response against parasitoid infection. EMBO reports, 2015. 16(12): p. 1664-72.

54. Gutierrez, E., et al., Specialized hepatocyte-like cells regulate Drosophila lipid metabolism. Nature, 2007. 445(7125): p. 275-80.

55. Dzierzak, E. and N.A. Speck, Of lineage and legacy: the development of mammalian hematopoietic stem cells. Nature immunology, 2008. 9(2): p. 129-36.

56. Gosselin, D., et al., Environment drives selection and function of enhancers controlling tissue-specific macrophage identities. Cell, 2014. 159(6): p. 1327-40.

57. Lavin, Y., et al., Tissue-resident macrophage enhancer landscapes are shaped by the local microenvironment. Cell, 2014. 159(6): p. 1312-26.

58. Suzuki, T., et al., Pulmonary macrophage transplantation therapy. Nature, 2014. 514(7523): p. 450-4.

59. Lebestky, T., S.H. Jung, and U. Banerjee, A Serrate-expressing signaling center controls Drosophila hematopoiesis. Genes Dev, 2003. 17(3): p. 348-53.

60. Jung, S.H., et al., The Drosophila lymph gland as a developmental model of hematopoiesis. Development, 2005. 132(11): p. 2521-33.

61. Mandal, L., U. Banerjee, and V. Hartenstein, Evidence for a fruit fly hemangioblast and similarities between lymph-gland hematopoiesis in fruit fly and mammal aorta-gonadalmesonephros mesoderm. Nat Genet, 2004. 36(9): p. 1019-23.

62. Zape, J.P. and A.C. Zovein, Hemogenic endothelium: origins, regulation, and implications for vascular biology. Seminars in cell \& developmental biology, 2011. 22(9): p. 1036-47.

63. Hirschi, K.K., Hemogenic endothelium during development and beyond. Blood, 2012. 119(21): p. 4823-7.

64. Martinez-Agosto, J.A., et al., The hematopoietic stem cell and its niche: a comparative view. Genes Dev, 2007. 21(23): p. 3044-60.

65. Krzemien, J., M. Crozatier, and A. Vincent, Ontogeny of the Drosophila larval hematopoietic organ, hemocyte homeostasis and the dedicated cellular immune response to parasitism. The International journal of developmental biology, 2010. 54(6-7): p. 1117-25.

66. Mandal, L., et al., A Hedgehog- and Antennapedia-dependent niche maintains Drosophila haematopoietic precursors. Nature, 2007. 446(7133): p. 320-4.

67. Krzemien, J., et al., Control of blood cell homeostasis in Drosophila larvae by the posterior signalling centre. Nature, 2007. 446(7133): p. 325-8.

68. Sinenko, S.A., et al., Dual role of wingless signaling in stem-like hematopoietic precursor maintenance in Drosophila. Dev Cell, 2009. 16(5): p. 756-63.

69. Dragojlovic-Munther, M. and J.A. Martinez-Agosto, Multifaceted roles of PTEN and TSC orchestrate growth and differentiation of Drosophila blood progenitors. Development, 2012. 139(20): p. 3752-63.

70. Krzemien, J., et al., Hematopoietic progenitors and hemocyte lineages in the Drosophila lymph gland. Developmental biology, 2010. 346(2): p. 310-9.

71. Davies, L.C., et al., Distinct bone marrow-derived and tissue-resident macrophage lineages proliferate at key stages during inflammation. Nature communications, 2013. 4: p. 1886.

72. Shi, C. and E.G. Pamer, Monocyte recruitment during infection and inflammation. Nature reviews. Immunology, 2011. 11(11): p. 762-74. 
73. Grigorian, M., L. Mandal, and V. Hartenstein, Hematopoiesis at the onset of metamorphosis: terminal differentiation and dissociation of the Drosophila lymph gland. Development genes and evolution, 2011. 221(3): p. 121-31.

74. Crozatier, M., et al., Cellular immune response to parasitization in Drosophila requires the EBF orthologue collier. PLoS Biol, 2004. 2(8): p. E196.

75. Pennetier, D., et al., Size control of the Drosophila hematopoietic niche by bone morphogenetic protein signaling reveals parallels with mammals. Proceedings of the National Academy of Sciences of the United States of America, 2012. 109(9): p. 3389-94.

76. Benmimoun, B., et al., The EBF transcription factor Collier directly promotes Drosophila blood cell progenitor maintenance independently of the niche. Proceedings of the National Academy of Sciences of the United States of America, 2015. 112(29): p. 9052-7.

77. Mondal, B.C., et al., Interaction between differentiating cell- and niche-derived signals in hematopoietic progenitor maintenance. Cell, 2011. 147(7): p. 1589-600.

78. Milton, C.C., et al., The Hippo pathway regulates hematopoiesis in Drosophila melanogaster. Current biology : CB, 2014. 24(22): p. 2673-80.

79. Ferguson, G.B. and J.A. Martinez-Agosto, Yorkie and Scalloped signaling regulates Notch-dependent lineage specification during Drosophila hematopoiesis. Current biology : CB, 2014. 24(22): p. 2665-72.

80. Myrick, K.V. and C.R. Dearolf, Hyperactivation of the Drosophila Hop jak kinase causes the preferential overexpression of eIF1A transcripts in larval blood cells. Gene, 2000. 244(1-2): p. 119-25.

81. Minakhina, S., W. Tan, and R. Steward, JAK/STAT and the GATA factor Pannier control hemocyte maturation and differentiation in Drosophila. Developmental biology, 2011. 352(2): p. 308-16.

82. Qiu, P., P.C. Pan, and S. Govind, A role for the Drosophila Toll/Cactus pathway in larval hematopoiesis. Development, 1998. 125(10): p. 1909-20.

83. Dragojlovic-Munther, M. and J.A. Martinez-Agosto, Extracellular matrix-modulated Heartless signaling in Drosophila blood progenitors regulates their differentiation via a Ras/ETS/FOG pathway and target of rapamycin function. Developmental biology, 2013. 384(2): p. 313-30.

84. Grigorian, M., et al., The proteoglycan Trol controls the architecture of the extracellular matrix and balances proliferation and differentiation of blood progenitors in the Drosophila lymph gland. Developmental biology, 2013. 384(2): p. 301-12.

85. Tokusumi, T., et al., Germ line differentiation factor Bag of Marbles is a regulator of hematopoietic progenitor maintenance during Drosophila hematopoiesis. Development, 2011. 138(18): p. 3879-84.

86. Tokusumi, T., et al., Bag of Marbles controls the size and organization of the Drosophila hematopoietic niche through interactions with the Insulin-like growth factor pathway and Retinoblastoma-family protein. Development, 2015. 142(13): p. 2261-7.

87. Minakhina, S., M. Druzhinina, and R. Steward, Zfrp8, the Drosophila ortholog of PDCD2, functions in lymph gland development and controls cell proliferation. Development, 2007. 134(13): p. 2387-96.

88. Remillieux-Leschelle, N., P. Santamaria, and N.B. Randsholt, Regulation of larval hematopoiesis in Drosophila melanogaster: a role for the multi sex combs gene. Genetics, 2002. 162(3): p. 1259-74. 
89. Holz, A., et al., The two origins of hemocytes in Drosophila. Development, 2003. 130(20): p. 4955-62.

90. Sampson, C.J., U. Amin, and J.P. Couso, Activation of Drosophila hemocyte motility by the ecdysone hormone. Biology open, 2013. 2(12): p. 1412-20.

91. Ayyaz, A., H. Li, and H. Jasper, Haemocytes control stem cell activity in the Drosophila intestine. Nature cell biology, 2015. 17(6): p. 736-48.

92. Ghosh, S., et al., Active hematopoietic hubs in Drosophila adults generate hemocytes and contribute to immune response. Developmental cell, 2015. 33(4): p. 478-88.

93. Nelliot, A., N. Bond, and D.K. Hoshizaki, Fat-body remodeling in Drosophila melanogaster. Genesis, 2006. 44(8): p. 396-400.

94. Elrod-Erickson, M., S. Mishra, and D. Schneider, Interactions between the cellular and humoral immune responses in Drosophila. Current biology : CB, 2000. 10(13): p. 781-4.

95. Dionne, M.S., N. Ghori, and D.S. Schneider, Drosophila melanogaster is a genetically tractable model host for Mycobacterium marinum. Infection and immunity, 2003. 71(6): p. 3540-50.

96. Akbar, M.A., et al., The full-of-bacteria gene is required for phagosome maturation during immune defense in Drosophila. The Journal of cell biology, 2011. 192(3): p. 38390.

97. Lanot, R., et al., Postembryonic hematopoiesis in Drosophila. Dev Biol, 2001. 230(2): p. 243-57.

98. Evans, C.J., T. Liu, and U. Banerjee, Drosophila hematopoiesis: markers and methods for molecular genetic analysis. Methods, 2014.

99. Clark, R.I., et al., Multiple TGF-beta superfamily signals modulate the adult Drosophila immune response. Current biology : CB, 2011. 21(19): p. 1672-7.

100. Bataille, L., et al., Resolving embryonic blood cell fate choice in Drosophila: interplay of GCM and RUNX factors. Development, 2005. 132(20): p. 4635-44.

101. Mackenzie, D.K., L.F. Bussiere, and M.C. Tinsley, Senescence of the cellular immune response in Drosophila melanogaster. Experimental gerontology, 2011. 46(11): p. 853-9.

102. Horn, L., J. Leips, and M. Starz-Gaiano, Phagocytic ability declines with age in adult Drosophila hemocytes. Aging cell, 2014. 13(4): p. 719-28.

103. Williams, M.J., Drosophila hemopoiesis and cellular immunity. J Immunol, 2007. 178(8): p. 4711-6.

104. Stuart, L.M. and R.A. Ezekowitz, Phagocytosis and comparative innate immunity: learning on the fly. Nature reviews. Immunology, 2008. 8(2): p. 131-41.

105. Franc, N.C., et al., Requirement for croquemort in phagocytosis of apoptotic cells in Drosophila. Science, 1999. 284(5422): p. 1991-4.

106. Tung, T.T., et al., Phosphatidylserine recognition and induction of apoptotic cell clearance by Drosophila engulfment receptor Draper. Journal of biochemistry, 2013. 153(5): p. 483-91.

107. Kuraishi, T., et al., Pretaporter, a Drosophila protein serving as a ligand for Draper in the phagocytosis of apoptotic cells. The EMBO journal, 2009. 28(24): p. 3868-78.

108. Kocks, C., et al., Eater, a transmembrane protein mediating phagocytosis of bacterial pathogens in Drosophila. Cell, 2005. 123(2): p. 335-46.

109. Kurucz, E., et al., Nimrod, a putative phagocytosis receptor with EGF repeats in Drosophila plasmatocytes. Current biology : CB, 2007. 17(7): p. 649-54. 
110. Somogyi, K., et al., Evolution of genes and repeats in the Nimrod superfamily. Molecular biology and evolution, 2008. 25(11): p. 2337-47.

111. Nagaosa, K., et al., Integrin betanu-mediated phagocytosis of apoptotic cells in Drosophila embryos. The Journal of biological chemistry, 2011. 286(29): p. 25770-7.

112. Zhou, L., et al., Programmed cell death in the Drosophila central nervous system midline. Curr Biol, 1995. 5(7): p. 784-90.

113. Sears, H.C., C.J. Kennedy, and P.A. Garrity, Macrophage-mediated corpse engulfment is required for normal Drosophila CNS morphogenesis. Development, 2003. 130(15): p. 3557-65.

114. Sonnenfeld, M.J. and J.R. Jacobs, Macrophages and glia participate in the removal of apoptotic neurons from the Drosophila embryonic nervous system. The Journal of comparative neurology, 1995. 359(4): p. 644-52.

115. Brückner, K., et al., The PDGF/VEGF Receptor controls blood cell survival in Drosophila. Dev Cell, 2004. 7.

116. Baer, M.M., et al., The role of apoptosis in shaping the tracheal system in the Drosophila embryo. Mechanisms of development, 2010. 127(1-2): p. 28-35.

117. Yasothornsrikul, S., et al., viking: identification and characterization of a second type IV collagen in Drosophila. Gene, 1997. 198(1-2): p. 17-25.

118. Mirre, C., et al., De novo expression of a type IV collagen gene in Drosophila embryos is restricted to mesodermal derivatives and occurs at germ band shortening. Development, 1988. 102(2): p. 369-76.

119. Kusche-Gullberg, M., et al., Laminin A chain: expression during Drosophila development and genomic sequence. The EMBO journal, 1992. 11(12): p. 4519-27.

120. Fogerty, F.J., et al., Tiggrin, a novel Drosophila extracellular matrix protein that functions as a ligand for Drosophila alpha PS2 beta PS integrins. Development, 1994. 120(7): p. 1747-58.

121. Kramerova, I.A., A.A. Kramerov, and J.H. Fessler, Alternative splicing of papilin and the diversity of Drosophila extracellular matrix during embryonic morphogenesis. Developmental dynamics : an official publication of the American Association of Anatomists, 2003. 226(4): p. 634-42.

122. Nelson, R.E., et al., Peroxidasin: a novel enzyme-matrix protein of Drosophila development. Embo J, 1994. 13(15): p. 3438-47.

123. Bhave, G., et al., Peroxidasin forms sulfilimine chemical bonds using hypohalous acids in tissue genesis. Nature chemical biology, 2012. 8(9): p. 784-90.

124. Comber, K., et al., A dual role for the betaPS integrin myospheroid in mediating Drosophila embryonic macrophage migration. Journal of cell science, 2013. 126(Pt 15): p. 3475-84.

125. Olofsson, B. and D.T. Page, Condensation of the central nervous system in embryonic Drosophila is inhibited by blocking hemocyte migration or neural activity. Dev Biol, 2005. 279(1): p. 233-43.

126. Bunt, S., et al., Hemocyte-secreted type IV collagen enhances BMP signaling to guide renal tubule morphogenesis in Drosophila. Developmental cell, 2010. 19(2): p. 296-306.

127. Siekhaus, D., et al., RhoL controls invasion and Rap1 localization during immune cell transmigration in Drosophila. Nature cell biology, 2010. 12(6): p. 605-10. 
128. Wood, W., C. Faria, and A. Jacinto, Distinct mechanisms regulate hemocyte chemotaxis during development and wound healing in Drosophila melanogaster. J Cell Biol, 2006. 173(3): p. 405-16.

129. Ratheesh, A., V. Belyaeva, and D.E. Siekhaus, Drosophila immune cell migration and adhesion during embryonic development and larval immune responses. Current opinion in cell biology, 2015. 36: p. 71-79.

130. Evans, I.R. and W. Wood, Drosophila blood cell chemotaxis. Current opinion in cell biology, 2014. 30: p. 1-8.

131. Wood, W. and A. Jacinto, Drosophila melanogaster embryonic haemocytes: masters of multitasking. Nat Rev Mol Cell Biol, 2007. 8(7): p. 542-51.

132. Razzell, W., W. Wood, and P. Martin, Swatting flies: modelling wound healing and inflammation in Drosophila. Disease models \& mechanisms, 2011. 4(5): p. 569-74.

133. Stramer, B.M. and M.S. Dionne, Unraveling tissue repair immune responses in flies. Seminars in immunology, 2014. 26(4): p. 310-4.

134. Wood, W., et al., Wound healing recapitulates morphogenesis in Drosophila embryos. Nat Cell Biol, 2002. 4(11): p. 907-12.

135. Brock, A.R., D.T. Babcock, and M.J. Galko, Active cop, passive cop: developmental stage-specific modes of wound-induced blood cell recruitment in Drosophila. Fly (Austin), 2008. 2(6): p. 303-5.

136. Vlisidou, I., et al., Drosophila embryos as model systems for monitoring bacterial infection in real time. PLoS pathogens, 2009. 5(7): p. e1000518.

137. Tan, K.L., I. Vlisidou, and W. Wood, Ecdysone mediates the development of immunity in the Drosophila embryo. Current biology : CB, 2014. 24(10): p. 1145-52.

138. Shim, J., et al., Olfactory control of blood progenitor maintenance. Cell, 2013. 155(5): p. 1141-53.

139. Shim, J., T. Mukherjee, and U. Banerjee, Direct sensing of systemic and nutritional signals by haematopoietic progenitors in Drosophila. Nature cell biology, 2012. 14(4): p. 394-400.

140. Tokusumi, Y., et al., Gene regulatory networks controlling hematopoietic progenitor niche cell production and differentiation in the Drosophila lymph gland. PloS one, 2012. 7(7): p. e41604.

141. Benmimoun, B., et al., Dual role for Insulin/TOR signaling in the control of hematopoietic progenitor maintenance in Drosophila. Development, 2012. 139(10): p. 1713-7.

142. Sinenko, S.A., J. Shim, and U. Banerjee, Oxidative stress in the haematopoietic niche regulates the cellular immune response in Drosophila. EMBO reports, 2012. 13(1): p. 83-9.

143. Owusu-Ansah, E. and U. Banerjee, Reactive oxygen species prime Drosophila haematopoietic progenitors for differentiation. Nature, 2009. 461(7263): p. 537-41.

144. Rizki, T.M. and R.M. Rizki, Lamellocyte differentiation in Drosophila larvae parasitized by Leptopilina. Dev Comp Immunol, 1992. 16(2-3): p. 103-10.

145. Small, C., et al., An introduction to parasitic wasps of Drosophila and the antiparasite immune response. Journal of visualized experiments : JoVE, 2012(63): p. e3347.

146. Sorrentino, R.P., Y. Carton, and S. Govind, Cellular immune response to parasite infection in the Drosophila lymph gland is developmentally regulated. Dev Biol, 2002. 243(1): p. 65-80. 
147. Nam, H.J., et al., Involvement of pro-phenoloxidase 3 in lamellocyte-mediated spontaneous melanization in Drosophila. Molecules and cells, 2008. 26(6): p. 606-10.

148. Vanha-Aho, L.M., et al., Edin Expression in the Fat Body Is Required in the Defense Against Parasitic Wasps in Drosophila melanogaster. PLoS pathogens, 2015. 11(5): p. e1004895.

149. Schmid, M.R., et al., Control of Drosophila blood cell activation via Toll signaling in the fat body. PloS one, 2014. 9(8): p. e102568.

150. Zettervall, C.J., et al., A directed screen for genes involved in Drosophila blood cell activation. Proc Natl Acad Sci U S A, 2004. 101(39): p. 14192-7.

151. Williams, M.J., et al., Rac1 signalling in the Drosophila larval cellular immune response. Journal of cell science, 2006. 119(Pt 10): p. 2015-24.

152. Ferrandon, D., J.L. Imler, and J.A. Hoffmann, Sensing infection in Drosophila: Toll and beyond. Seminars in immunology, 2004. 16(1): p. 43-53.

153. Nehme, N.T., et al., A model of bacterial intestinal infections in Drosophila melanogaster. PLoS pathogens, 2007. 3(11): p. e173.

154. Basset, A., et al., The phytopathogenic bacteria Erwinia carotovora infects Drosophila and activates an immune response. Proceedings of the National Academy of Sciences of the United States of America, 2000. 97(7): p. 3376-81.

155. Foley, E. and P.H. O'Farrell, Nitric oxide contributes to induction of innate immune responses to gram-negative bacteria in Drosophila. Genes \& development, 2003. 17(1): p. $115-25$.

156. Wu, S.C., et al., Infection-induced intestinal oxidative stress triggers organ-to-organ immunological communication in Drosophila. Cell host \& microbe, 2012. 11(4): p. 410-7.

157. Brennan, C.A., et al., Psidin is required in Drosophila blood cells for both phagocytic degradation and immune activation of the fat body. Current biology : CB, 2007. 17(1): p. 67-72.

158. Akhouayri, I., et al., Toll-8/Tollo negatively regulates antimicrobial response in the Drosophila respiratory epithelium. PLoS pathogens, 2011. 7(10): p. e1002319.

159. Lemaitre, B. and J. Hoffmann, The host defense of Drosophila melanogaster. Annu Rev Immunol, 2007. 25: p. 697-743.

160. Hoffmann, J.A. and J.M. Reichhart, Drosophila innate immunity: an evolutionary perspective. Nat Immunol, 2002. 3(2): p. 121-6.

161. Neyen, C., et al., Methods to study Drosophila immunity. Methods, 2014. 68(1): p. 11628.

162. Shia, A.K., et al., Toll-dependent antimicrobial responses in Drosophila larval fat body require Spatzle secreted by haemocytes. Journal of cell science, 2009. 122(Pt 24): p. 4505-15.

163. Pearson, A.M., et al., Identification of cytoskeletal regulatory proteins required for efficient phagocytosis in Drosophila. Microbes Infect, 2003. 5(10): p. 815-24.

164. Markus, R., et al., Sterile wounding is a minimal and sufficient trigger for a cellular immune response in Drosophila melanogaster. Immunology letters, 2005. 101(1): p. 10811.

165. Babcock, D.T., et al., Circulating blood cells function as a surveillance system for damaged tissue in Drosophila larvae. Proc Natl Acad Sci U S A, 2008. 105(29): p. 10017-22. 
166. Cohen, S.M., Imaginal disc development. The Development of Drosophila melanogaster Bate M, Martinez Arias A, eds. , 1993. 2: p. 747-841.

167. Williams, D.W. and J.W. Truman, Cellular mechanisms of dendrite pruning in Drosophila: insights from in vivo time-lapse of remodeling dendritic arborizing sensory neurons. Development, 2005. 132(16): p. 3631-42.

168. Han, C., et al., Epidermal cells are the primary phagocytes in the fragmentation and clearance of degenerating dendrites in Drosophila. Neuron, 2014. 81(3): p. 544-60.

169. Doherty, J., et al., Ensheathing glia function as phagocytes in the adult Drosophila brain. The Journal of neuroscience : the official journal of the Society for Neuroscience, 2009. 29(15): p. 4768-81.

170. Kiger, J.A., Jr., J.E. Natzle, and M.M. Green, Hemocytes are essential for wing maturation in Drosophila melanogaster. Proc Natl Acad Sci U S A, 2001. 98(18): p. 10190-5.

171. Moreira, C.G., A. Jacinto, and S. Prag, Drosophila integrin adhesion complexes are essential for hemocyte migration in vivo. Biology open, 2013. 2(8): p. 795-801.

172. Sander, M., et al., Drosophila pupal macrophages--a versatile tool for combined ex vivo and in vivo imaging of actin dynamics at high resolution. European journal of cell biology, 2013. 92(10-11): p. 349-54.

173. Regan, J.C., et al., Steroid hormone signaling is essential to regulate innate immune cells and fight bacterial infection in Drosophila. PLoS pathogens, 2013. 9(10): p. e1003720.

174. Defaye, A., et al., Genetic ablation of Drosophila phagocytes reveals their contribution to both development and resistance to bacterial infection. Journal of innate immunity, 2009. 1(4): p. 322-34.

175. Charroux, B. and J. Royet, Elimination of plasmatocytes by targeted apoptosis reveals their role in multiple aspects of the Drosophila immune response. Proc Natl Acad Sci U S A, 2009. 106(24): p. 9797-802.

176. Arefin, B., et al., Apoptosis in Hemocytes Induces a Shift in Effector Mechanisms in the Drosophila Immune System and Leads to a Pro-Inflammatory State. PloS one, 2015. 10(8): p. e0136593.

177. Ramet, M., et al., Functional genomic analysis of phagocytosis and identification of a Drosophila receptor for E. coli. Nature, 2002. 416(6881): p. 644-8.

178. Losick, V.P., D.T. Fox, and A.C. Spradling, Polyploidization and cell fusion contribute to wound healing in the adult Drosophila epithelium. Current biology : CB, 2013. 23(22): p. 2224-32.

179. Soares, L., M. Parisi, and N.M. Bonini, Axon injury and regeneration in the adult Drosophila. Scientific reports, 2014. 4: p. 6199.

180. King, J.G. and J.F. Hillyer, Infection-induced interaction between the mosquito circulatory and immune systems. PLoS pathogens, 2012. 8(11): p. e1003058.

181. Nutting, W.L., A comparative anatomical study of the heart and accessory structures of the orthopteroid insects. J. Morphol., 1951. 89: p. 501-98.

182. Jones, T.F., Hemocytopoiesis in insects. Regulation of Hematopoiesis A.S. Gordon (ed.), 1970: p. 7-65.

183. Wigglesworth, V.B., Insect blood cells. Annu. Rev. Entomol., 1959. 4: p. 1-16.

184. Agaisse, H., et al., Signaling Role of Hemocytes in Drosophila JAK/STAT-Dependent Response to Septic Injury. Dev Cell, 2003. 5(3): p. 441-50. 
185. Tzou, P., et al., Tissue-specific inducible expression of antimicrobial peptide genes in Drosophila surface epithelia. Immunity, 2000. 13(5): p. 737-48.

186. Ferrandon, D., et al., A drosomycin-GFP reporter transgene reveals a local immune response in Drosophila that is not dependent on the Toll pathway. The EMBO journal, 1998. 17(5): p. 1217-27.

187. Apidianakis, Y. and L.G. Rahme, Drosophila melanogaster as a model host for studying Pseudomonas aeruginosa infection. Nature protocols, 2009. 4(9): p. 1285-94.

188. Pham, L.N., et al., A specific primed immune response in Drosophila is dependent on phagocytes. PLoS Pathog, 2007. 3(3): p. e26.

189. Nehme, N.T., et al., Relative roles of the cellular and humoral responses in the Drosophila host defense against three gram-positive bacterial infections. PloS one, 2011. 6(3): p. e14743.

190. Avet-Rochex, A., et al., Suppression of Drosophila cellular immunity by directed expression of the ExoS toxin GAP domain of Pseudomonas aeruginosa. Cellular microbiology, 2005. 7(6): p. 799-810.

191. Bergeret, E., et al., TM9SF4 is required for Drosophila cellular immunity via cell adhesion and phagocytosis. Journal of cell science, 2008. 121(Pt 20): p. 3325-34.

192. Braun, A., J.A. Hoffmann, and M. Meister, Analysis of the Drosophila host defense in domino mutant larvae, which are devoid of hemocytes. Proc Natl Acad Sci U S A, 1998. 95(24): p. 14337-42.

193. Stone, E.F., et al., The circadian clock protein timeless regulates phagocytosis of bacteria in Drosophila. PLoS pathogens, 2012. 8(1): p. e1002445.

194. Watson, F.L., et al., Extensive diversity of Ig-superfamily proteins in the immune system of insects. Science, 2005. 309(5742): p. 1874-8.

195. Hauton, C. and V.J. Smith, Adaptive immunity in invertebrates: a straw house without a mechanistic foundation. BioEssays : news and reviews in molecular, cellular and developmental biology, 2007. 29(11): p. 1138-46.

196. Chazaud, B., Macrophages: supportive cells for tissue repair and regeneration. Immunobiology, 2014. 219(3): p. 172-8.

197. Okabe, Y. and R. Medzhitov, Tissue biology perspective on macrophages. Nature immunology, 2015. 17(1): p. 9-17.

198. Wang, X., et al., Increased infiltrated macrophages in benign prostatic hyperplasia $(B P H)$ : role of stromal androgen receptor in macrophage-induced prostate stromal cell proliferation. The Journal of biological chemistry, 2012. 287(22): p. 18376-85.

199. Kamo, T., H. Akazawa, and I. Komuro, Cardiac nonmyocytes in the hub of cardiac hypertrophy. Circulation research, 2015. 117(1): p. 89-98.

200. Kelsey, E.M., et al., Schnurri regulates hemocyte function to promote tissue recovery after DNA damage. Journal of cell science, 2012. 125(Pt 6): p. 1393-400.

201. Fogarty, E.F., et al., Extracellular Reactive Oxygen Species Drive Apoptosis-Induced Proliferation via Drosophila Macrophages. Curr Biol, 2016. 26: p. http://dx.doi.org/10.1016/j.cub.2015.12.064.

202. Buchon, N., et al., Invasive and indigenous microbiota impact intestinal stem cell activity through multiple pathways in Drosophila. Genes \& development, 2009. 23(19): p. 233344.

203. Biteau, B., et al., Lifespan extension by preserving proliferative homeostasis in Drosophila. PLoS genetics, 2010. 6(10): p. e1001159. 
204. Ayyaz, A. and H. Jasper, Intestinal inflammation and stem cell homeostasis in aging Drosophila melanogaster. Frontiers in cellular and infection microbiology, 2013. 3: p. 98.

205. Christofi, T. and Y. Apidianakis, Drosophila and the hallmarks of cancer. Advances in biochemical engineering/biotechnology, 2013. 135: p. 79-110.

206. Tipping, M. and N. Perrimon, Drosophila as a model for context-dependent tumorigenesis. Journal of cellular physiology, 2014. 229(1): p. 27-33.

207. Hauling, T., et al., A Drosophila immune response against Ras-induced overgrowth. Biology open, 2014. 3(4): p. 250-60.

208. Pastor-Pareja, J.C., M. Wu, and T. Xu, An innate immune response of blood cells to tumors and tissue damage in Drosophila. Disease models \& mechanisms, 2008. 1(2-3): p. 144-54; discussion 153.

209. Cordero, J.B., et al., Oncogenic Ras diverts a host TNF tumor suppressor activity into tumor promoter. Developmental cell, 2010. 18(6): p. 999-1011.

210. Parisi, F., et al., Transformed epithelia trigger non-tissue-autonomous tumor suppressor response by adipocytes via activation of Toll and Eiger/TNF signaling. Cell reports, 2014. 6(5): p. 855-67.

211. Siveen, K.S. and G. Kuttan, Role of macrophages in tumour progression. Immunology letters, 2009. 123(2): p. 97-102.

212. Mantovani, A., et al., Cancer-related inflammation. Nature, 2008. 454(7203): p. 436-44.

213. Sica, A., P. Allavena, and A. Mantovani, Cancer related inflammation: the macrophage connection. Cancer letters, 2008. 267(2): p. 204-15.

214. Apidianakis, Y., et al., Synergy between bacterial infection and genetic predisposition in intestinal dysplasia. Proceedings of the National Academy of Sciences of the United States of America, 2009. 106(49): p. 20883-8.

215. Bangi, E., et al., Immune response to bacteria induces dissemination of Ras-activated Drosophila hindgut cells. EMBO reports, 2012. 13(6): p. 569-76.

\section{Figure Legends}

Figure 1. Ontogeny of macrophages in Drosophila and mouse development

(A) Two waves of hematopoiesis during Drosophila development. The embryonic/larval lineage (in red) originates from the head mesoderm (HM) of the embryo, differentiates in the embryo, and subsequently expands in the larva as self-renewing tissue macrophages (plasmatocytes). The progenitor-based Lymph Gland lineage (in blue) originates in the embryo and differentiates in the late larva. Macrophages of both lineages persist through pupal development into the adult. 
(B) Three waves of hematopoiesis during mouse development. The primitive wave (in green) emerges in the yolk sac and gives rise to the earliest macrophages; this lineage does not persist after birth. The wave of erythro-myeloid progenitors (EMPs, in red) also emerges in the yolk sac. These cells mature in the fetal liver, and colonize local microenvironments in various organs as tissue-resident macrophages that self-renew and persist. The definitive hematopoietic wave emerges from hemogenic endothelium (major arteries) that give rise to hematopoietic stem cells (HSCs in blue), which colonize the fetal liver and later the bone marrow, giving rise to the monocyte lineage of macrophages.

\section{Figure 2. Blood cell lineages in Drosophila}

(A) The embryonic lineage of hemocytes (blood cells) with parallels to self-renewing tissue macrophages in vertebrates. Prohemocyte progenitors (blue) originate in the embryo and differentiate into plasmatocytes (macrophages, red) and a small number of crystal cells (orange); plasmatocytes are quiescent (q) until the end of embryogenesis. In the larva, plasmatocytes colonize local microenvironments, in particular the Hematopoietic Pockets, and expand by selfrenewal. Plasmatocytes also give rise to a small number of crystal cells, and, upon immune challenge, lamellocytes (purple).

(B) Lymph Gland hematopoiesis with parallels to progenitor-based hematopoiesis in vertebrates. Lymph Gland prohemocytes (blue) are specified from the cardiogenic mesoderm of the embryo. They proliferate at a low rate until the 2nd larval instar, then start differentiating, forming (1) intermediate progenitors and plasmatocytes (red), which expand further by proliferation; (2) crystal cells (orange); (3) lamellocytes (purple). The primary lobe of the differentiating Lymph 
Gland is organized into a medullary zone (MZ) of quiescent (q) progenitors, and a cortical zone (CZ) of differentiating hemocytes; differentiation of progenitors is completed by $12 \mathrm{~h}$ after puparium formation.

Both lineages of hemocytes are mobilized in the pupa and persist into the adult, where new blood

cell production subsides and hemocyte numbers decline. Plasmatocytes and small numbers of crystal cells, but no lamellocytes, are present in the adult.

\section{Figure 3 Innate immune responses in Drosophila}

Throughout its life cycle, Drosophila can mount cellular and humoral innate immune responses. Cellular immune responses involve phagoctytosis by plasmatocytes, melanization by crystal cells and lamellocytes, and encapsulation by lamellocytes. Humoral responses involve the induction of antimicrobial peptide (AMP) expression in a number of tissues. In the larva, immune responses include the mobilization of resident plasmatocytes and their differentiation into lamellocytes, and the precocious differentiation and mobilization of Lymph Gland hemocytes. In the adult, hemocytes reside in proximity to tissues of innate immunity and barrier epithelia, such as fat body (brown), respiratory epithelia (purple), gastrointestinal system (teal) and circulatory system (gray). 


\section{Finu ure 1}

\section{A Hematopoietic waves in Drosophila development}

\section{Lymph Gland lineage}

Embryonic/ larval lineage

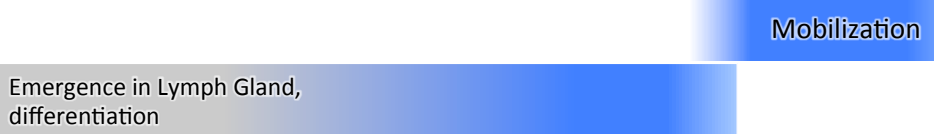

Mobilization

'Tissue Macrophages'

self-renewal in Hematopoietic Pockets; differentiation

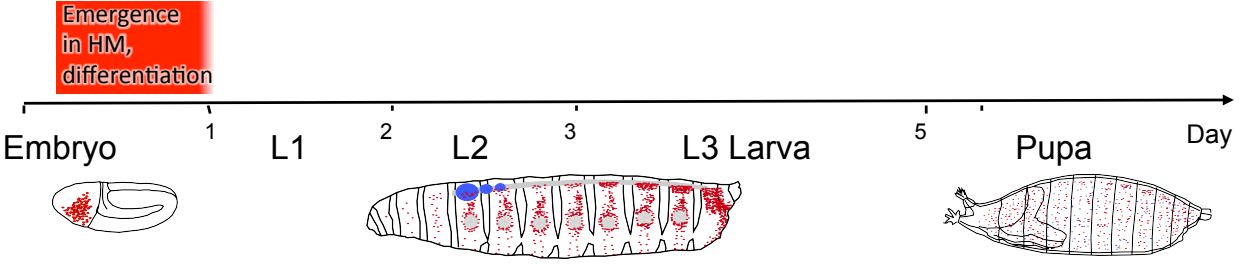

Hematopoietic waves in mouse development

B

Differentiation in

bone marrow

\section{Hematopoietic Stem Cell} HSC

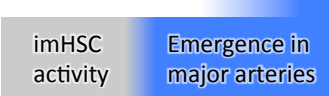

Erythro-Myeloid

Progenitor

EMP

\section{Differentiation in fetal liver}

Emergence in yolk sac

Primitive

Differentiation in bloodstream

Wave 
Finı 2

Embryonic Lineage

"Self-renewing tissue macrophage"

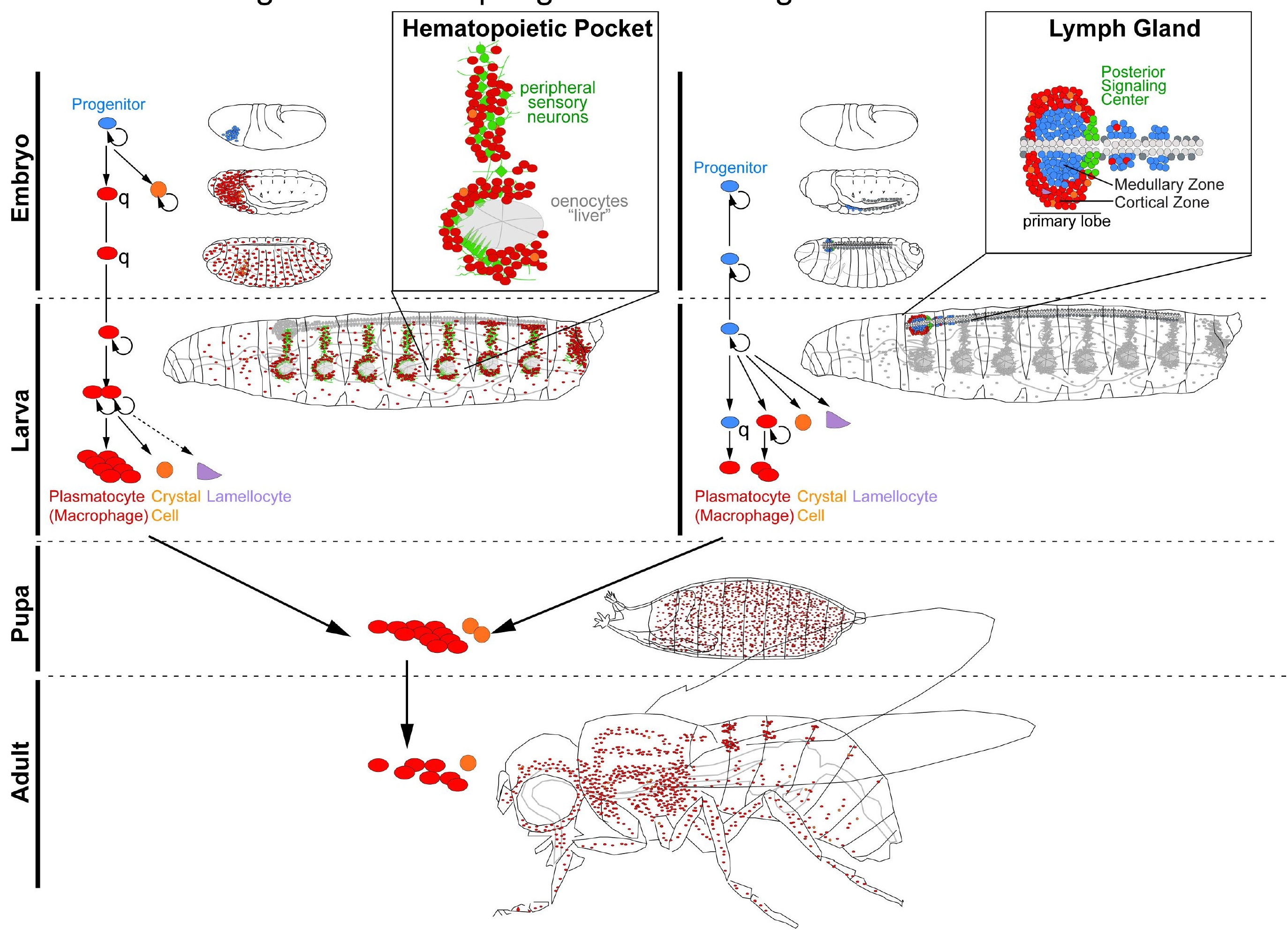

Lymph Gland Lineage "Progenitor-based"

Plasmatocyte Crystal Lamellocyte

(Macrophage) Cel 
Finure 3

\begin{tabular}{|c|c|}
\hline \multicolumn{2}{|c|}{ Immune Response Larva } \\
\hline Cellular response & Humoral response \\
\hline Phagocytosis, encapsulation, melanization & AMP Synthesis \\
\hline \multicolumn{2}{|l|}{ Hematopoietic Pockets } \\
\hline \multicolumn{2}{|l|}{ Mobilization } \\
\hline \multicolumn{2}{|l|}{ Lamellocyte differentiation } \\
\hline $\begin{array}{l}\text { Lymph Gland } \\
\text { Precocious differentiation } \\
\text { Mobilization }\end{array}$ & 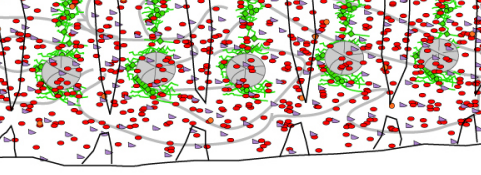 \\
\hline
\end{tabular}

Immune Response

\section{Embryo}

Cellular response

Phagocytosis, melanization

Humoral response

AMP Synthesis
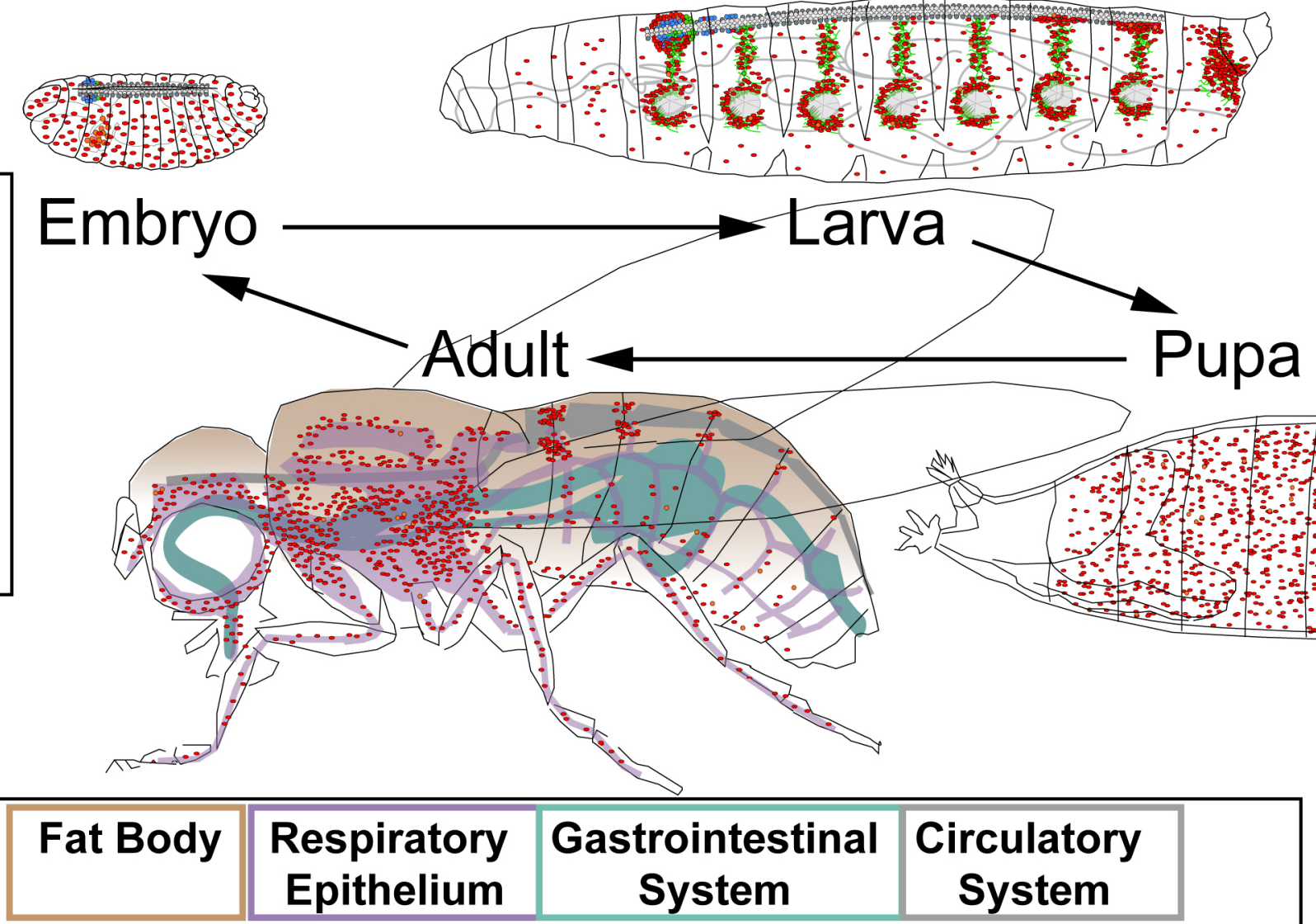

Cellular response

Phagocytosis, melanization
Humoral response

AMP Synthesis

Immune Response Adult 